\title{
Concept and design of charged particle optics using energy Fourier plane collimation
}

\author{
Yang Guojun, Wei Tao, Zhang Zhuo, He Xiaozhong, Zhang Xiaoding, Li Yiding, and Shi Jinshui \\ Institute of Fluid Physics, CAEP, Mianyang, Sichuan 621900, China
}

(Received 28 March 2014; published 22 September 2014)

\begin{abstract}
Charged particle radiography has become a promising new approach in the field of transmission radiography because of the invention of the magnetic imaging lens. The using of the imaging lens makes it possible for thick objects to get significantly improved transmission radiography. Currently, the conventional charged particle radiography only uses the information of the flux attenuation and the angular scattering of the transmitted particles to determine the properties of the sample. However, the energy loss of the incident particles introduced by ionizations throughout the object limits the spatial resolution of the image because of the chromatic blur. In this paper a new concept of imaging lens that uses the information of the energy loss is proposed. With a specially designed imaging lens, the information of the energy loss could result in apparent contrast in the final image. This design procedure of the energy loss imaging lens is presented, and a preliminary design is verified by numerical simulations. Experimental demonstration is also expected on a cyclotron at the Institute of Fluid Physics, CAEP.
\end{abstract}

DOI: 10.1103/PhysRevSTAB.17.094701

PACS numbers: 41.85.-p

\section{INTRODUCTION}

Charged particles are useful tools for transmission radiography. The early charged particle radiography typically involves incident radiation such as protons or electrons, a portion of which is transmitted through an object of interest to a detector plane forming a shadow graph there. The concept of the range-edge proton radiography was proposed by Koehler in 1968 [1]. Subsequently, Koehler and others [2-5] demonstrated that the high-contrast images obtained by proton radiography can provide improved imaging of low contrast lesions in human specimens over conventional x-ray techniques. Hanson described a proton CT method in 1981 [6,7], in which projections necessary for computer tomography (CT) reconstructions were obtained by measuring the proton energy losses. In recent years, research works $[8,9]$ are driven by the need of using the therapeutic proton beam for CT imaging.

Information contained in the energy loss was adopted in all the works mentioned above. However, the multiple Coulomb scattering (MCS) effect will seriously blur the final image. The magnetic imaging lens, which can focus the scattered particles, was suggested by Zumbro and Mottershead in order to improve the quality of the image [10]. The so-called Zumbro lens has also been successfully demonstrated both by simulations and experiments [11-14].

baita00@aliyun.com

Published by the American Physical Society under the terms of the Creative Commons Attribution 3.0 License. Further distribution of this work must maintain attribution to the author(s) and the published article's title, journal citation, and DOI.
Nuclear interaction with the nucleus [15], MCS with the Coulomb field of the nucleus [16], and ionization with the electrons [17] are three major types of interactions occurring when protons pass through materials. They will result in flux attenuation, angular scattering, and energy loss of protons, respectively. The effect of interactions may be somewhat different for electrons or heavy ions, and the following description is subject to the case of protons.

By using the radiography system with Zumbro lens, the sample properties can be obtained from the information of the flux attenuation and the angular scattering, while the energy loss has been a handicap due to the chromatic blurring effect. In this paper, a new type of magnetic lens system, which also uses the information of the energy loss, is suggested.

\section{PRINCIPLE OF THE ZUMBRO LENS [10]}

To the first order, the transfer matrix $R$ of a charged particle transport system maps a particle with initial transverse coordinates $\left(x_{0}, \theta_{0}\right)$ to a final transverse position

$$
x=R_{11} x_{0}+R_{12} \theta_{0} .
$$

In order to obtain the point-to-point imaging, one gets

$$
R_{12}=R_{34}=0 .
$$

A Zumbro lens is composed of four identical quadrupole magnets, as shown in Fig. 1.

Under certain conditions [10], the transfer matrix of a Zumbro lens becomes the minus-identity $(-I)$ matrix, which satisfies the requirements for the point-to-point imaging. 


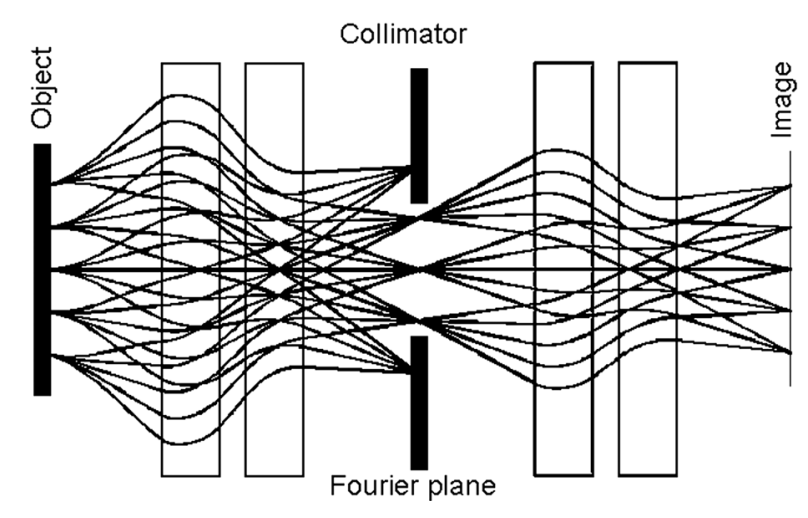

FIG. 1. Principle of magnetic lens.

The point-to-point imaging assumes a monoenergetic incident particles beam. However, when energy spread exists, the particles will deviate from the central trajectory due to the chromatic aberration. This problem can be solved by the second order chromatic matching design.

Suppose an incident particle comes from a virtual point source at a distance $L$ upstream of the entry plane of the lens. Because of both the MCS in the object and the nonzero emittance of the beam, the particle will exit the object at an angle

$$
\theta_{0}=w x_{0}+\phi,
$$

where $w\left(=L^{-1}\right)$ is the beam correlation coefficient, and $\varphi$ is the angular deviation from the ideal position-angle correlation line. The matrix elements in general are functions of the fractional momentum deviation $\Delta(\equiv \delta p / p)$. By expanding the final position of the particle in Taylor series form to the first order, it is found that $w$ should satisfy the following equations in order to realize the second-order chromatic matching design (primes indicate momentum derivatives)

$$
R_{11}^{\prime}+w R_{12}^{\prime}=0 .
$$

All positions depend on chromatic aberrations vanishing, and the final transverse position is then given by

$$
x=-x_{0}+R_{12}^{\prime} \phi \Delta .
$$

The remaining chromatic aberration only depends on the deviation angle, and the chromatic blur caused by the energy spread becomes $R_{12}^{\prime} \varphi \Delta$.

At the midplane of a chromatically matched identity lens, the transverse positions of a particles is given by

$$
x_{\text {mid }}=M_{12} \phi,
$$

where $M$ is the transfer matrix of the first half of the Zumbro lens. Equation (6) implies that after the first cell, the transverse position of the particle depends only on $\varphi$, not on $x$. In other words, the trajectories of the particles are completely sorted by angle $\varphi$ at the midplane. This plane is called angular Fourier plane, at which the angular collimator can be used.

\section{CONCEPT OF ENERGY FOURIER PLANE}

The concept of a Fourier plane has been widely used in geometry optics [18] and physical optics, e.g., synchrotron radiation optics [19].

With a Zumbro lens, thanks to the point-to-point imaging property and the existence of an angular Fourier plane, both nuclear attenuation and angular scattering can be used to generate contrast in the image, while with the energy-loss effect, as a dominate source of image blur which fundamentally limits the resolution of the system, has been left aside. However, it is found that the energy loss can also help to generate contrast in the radiography image, if the imaging lens is designed properly. Similar to the design of the Zumbro lens, an energy Fourier plane (EFP), at which the particles are horizontally sorted by their energies, can be found. At the EFP, a collimator only allows particles within a specific energy range to pass through and form an image at the image plane of the lens system.

The goal of this work is to design a new magnetic lens system, which provides point-to-point imaging in space, and an EFP which helps to realize the function of energy sorting at the same time.

\section{DESIGN METHOD FOR AN IMAGING SYSTEM WITH EFP}

The principle of EFP demands the existence of dispersion. So in an energy-loss imaging lens system, except for quadrupole magnets, transport elements with dispersion should be introduced, of which the simplest one is the dipole magnet, also called bending magnet.

However, a large dispersion will cause serious blur. Therefore the lens should be achromatic as an ensemble.

\section{A. Achromatic transport system [20]}

A traditional achromatic transport system, which consists of two identical dipole magnets and some quadrupole magnets, can be either symmetric or asymmetric. Figure 2 shows the sketch of a symmetry achromat.

Only symmetrical structure is used in this paper. Similar results may be obtained with an asymmetrical structure.

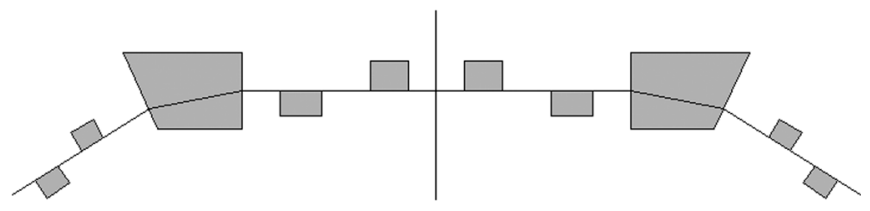

FIG. 2. Sketch of a symmetrical achromatic system. The trapezoids indicate bending magnets, and the rectangles indicate quadrupoles. 
Here mainly the bending plane with dispersion, the horizontal plane, or the $X$ plane, is considered. Without dispersion, the matrices in the vertical plane, also called the $Y$ plane, is an equivalent one with the sixth dimension element omitted.

Suppose the transfer matrix of the first half is $A$, and that of the second half is $B$.

$$
A=\left[\begin{array}{ccc}
A_{11} & A_{12} & A_{16} \\
A_{21} & A_{22} & A_{26} \\
0 & 0 & 1
\end{array}\right]
$$

and

$$
B=\left[\begin{array}{ccc}
A_{22} & A_{12} & A_{12} A_{26}-A_{16} A_{22} \\
A_{21} & A_{11} & A_{11} A_{26}-A_{16} A_{21} \\
0 & 0 & 1
\end{array}\right]
$$

The transfer matrix of whole lens, $R$, is then given by

$R=B \cdot A=\left[\begin{array}{ccc}1+2 A_{12} A_{21} & 2 A_{12} A_{22} & 2 A_{12} A_{26} \\ 2 A_{11} A_{21} & 1+2 A_{12} A_{21} & 2 A_{11} A_{26} \\ 0 & 0 & 1\end{array}\right]$

The achromatic requirement of the lens system forces the $(1,3)$ and $(2,3)$ elements of the above matrix $R$ to be zero, which leads to the following condition:

$$
A_{26}=0 .
$$

\section{B. Imaging condition}

According to Eqs. (2) and (9), the most convenient way to image in the $X$ plane is to set

$$
A_{12}=0 .
$$

Similarly, imaging in the $Y$ plane can be obtained by setting

$$
A_{34}=0 .
$$

Equations (11) and (12) suggest that the first half of the achromatic lens is itself an imaging lens. However, some of its characteristics have prevented it from taking the part of imaging lens by itself, including (a) chromatic and (b) difficulties in manipulation of the image magnifications $A_{11}$ and $A_{33}$.

\section{Existence condition of EFP}

Suppose that the transfer matrix describing the transportation of particles from the initial position to the EFP is $T$, the transverse position of a particle at the EFP is given by

$$
x_{\mathrm{EFP}}=T_{11} x_{0}+T_{12} \theta_{0}+T_{16} \delta .
$$

Similar to Eq. (6) for the angular Fourier plane, the condition for the EFP can be obtained as

$$
x_{\text {Fourier }}=T_{16} \delta \text {. }
$$

Equation (14) will be strictly satisfied if we have $T_{11}=T_{12}=0$. But it is impossible for $T_{11}$ and $T_{12}$ to be zero simultaneously since the determinant of $T$ cannot be zero.

However, a compromised solution can be found, if the beam incidents are in parallel, i.e., the correlation coefficient $w$ in Eq. (3) satisfies

$$
w=0 .
$$

If the angular spread is omitted, i.e., $\theta_{0}=\varphi=0$, only the following condition is required:

$$
T_{11}=0 \text {. }
$$

\section{The condition of the second-order chromatic matching}

The condition for the second-order chromatic matching is also needed.

Substituting Eq. (11) into the derivative of Eq. (9), we have

$$
R_{11}^{\prime}=2 A_{12}^{\prime} A_{21} .
$$

If the following condition is satisfied,

$$
A_{21}=0,
$$

one gets $R_{11}^{\prime}=0$. Equation (4) is now satisfied according to Eqs. (15) and (17)-(18).

Notice that now the transfer matrix (9) becomes the identity matrix $I$.

Similarly, the second-order chromatic matching in the $Y$ plane can be derived as

$$
A_{43}=0 .
$$

\section{E. Location of the EFP}

It is obvious that the midplane of the whole lens is not the EFP. Otherwise the determinant of the transfer matrix $T$ will be zero. [See Eqs. (11) and (16), and consider the fact that the transfer matrix $T$ becomes $A$.]

The following analysis will demonstrate that the midplanes of the bending magnets are the ideal EFPs.

In the first half of the lens, suppose the transfer matrices before and after the bending magnet are $T_{1}$ and $T_{2}$, respectively, and the transfer matrix of half of the bending magnet is $T_{h b}$ : 


$$
\begin{gathered}
T_{1}=\left[\begin{array}{lll}
a & b & 0 \\
c & d & 0 \\
0 & 0 & 1
\end{array}\right], \\
T_{2}=\left[\begin{array}{lll}
e & f & 0 \\
g & h & 0 \\
0 & 0 & 1
\end{array}\right], \\
T_{h b}=\left[\begin{array}{ccc}
\cos \frac{\phi}{2} & R \sin \frac{\phi}{2} & R\left(1-\cos \frac{\phi}{2}\right) \\
-\frac{1}{R} \sin \frac{\phi}{2} & \cos \frac{\phi}{2} & \sin \frac{\phi}{2} \\
0 & 0 & 1
\end{array}\right],
\end{gathered}
$$

where $a, b, c, d, e, f, g, h$ are unknowns, $R$ is the bending radius of the dipole magnet, and $\phi$ is the bending angle.

The transfer matrix of the first half of the lens system is given by

$$
A=T_{2} \cdot T_{h b} \cdot T_{h b} \cdot T_{1} .
$$

The transfer matrix from the beginning of the lens to the midplane of the first bending magnet is given by

$$
T=T_{h b} \cdot T_{1} .
$$

If conditions (10) and (18) are satisfied, i.e., $A_{26}=0$, $A_{21}=0, c$ in expression (20) and $h$ in expression (21) can be solved from expression (23). After a great deal of manipulation, $T_{11}=0$ is obtained, which is just the condition (16). It implies that the midplane of the first bending magnet is the right EFP we are looking for. The midplane of the second bending magnet can be demonstrated to be another EFP in a similar way.

As a matter of fact, no additional conditions are needed in this section.

\section{F. Improvement of the design}

In the previous section it was demonstrated that the midplanes of the bending magnets are the EFPs. However, the chromatic aberration at this plane, which is calculated to be only of a few micrometers in some cases, is too small to be made any use of.
For the convenience of beam manipulation, the chromatic aberration should be large enough. An effective way is to divide the bending magnet into two identical parts, separated by a drift space. It is easy to prove that the midplane between the two parts is still an EFP.

In practice, the angular spread of the incident beam cannot be omitted, and the position of a particle at the EFP is given by

$$
x_{\mathrm{EFP}}=T_{12} \varphi+T_{16} \delta .
$$

The influences of the angular spread and the energy spread cannot always be separated. Therefore the coefficient $T_{12}$ should be kept as small as possible. An effective method for minimizing $T_{12}$ is to put quadrupole magnets between the two dipole magnet parts. Following the procedure in the previous section, one can strictly prove that if these quadrupole magnets are symmetric about the midplane of the two dipole magnet parts, the midplane will remain an EFP.

\section{G. Brief summary of the design method}

In this section, design of a symmetric achromatic beam line was discussed; dispersion introduced by the dipole magnets was used to form the EFPs. If the transfer matrix $A$ of the first half of the beam line satisfies the following five conditions, i.e., $A_{26}=0, A_{12}=0, A_{21}=0, A_{34}=0$, and $A_{43}=0$, the beam line is proved to be both point-to-point imaging and achromatic. Furthermore, the midplanes of the bending magnets are strictly the EFPs with a parallel incident beam.

To further improve the performance, the bending magnets can be divided into two identical parts. The EFP will remain at the midplane between the two magnet parts if quadrupole magnets and drift spaces between them are designed properly.

The design procedure is roughly accomplished now. Modifications and improvements are continuing.

\section{A PRELIMINARY DESIGN OF THE ENERGY-LOSS IMAGING LENS}

An energy-loss imaging lens was designed for a $35 \mathrm{MeV}$ incident proton beam. The computer code Transport [21] was used for the beam dynamics design and the

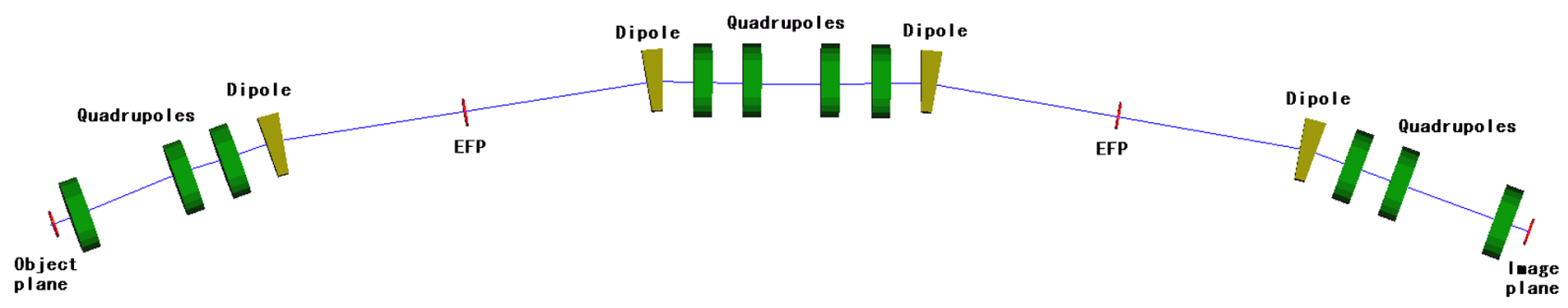

FIG. 3. G4beamline model of an energy-loss imaging lens designed. 
TABLE I. Parameters of the system shown in Fig. 3, expected for future validation.

\begin{tabular}{lccc}
\hline \hline Parameter & Value & Parameter & Value \\
\hline $\mathrm{T}_{12}$ (at the EFP) & $1.4 \mathrm{~mm} / \mathrm{mrad}$ & $\mathrm{T}_{16}$ (At the EFP) & $0.554 \mathrm{~m}$ \\
$\mathrm{~T}_{126}$ (at the image plane) & $-18.22 \mathrm{~m}$ & $\mathrm{~T}_{346}$ (at the image plane) & $-4.919 \mathrm{~m}$ \\
Bending radius of dipoles & $1.5 \mathrm{~m}$ & Bending angle of dipoles & $10 \mathrm{deg}$ \\
Quadrupole length & $0.3 \mathrm{~m}$ & Quadruple gradients (first five) & $-0.508761,0.298810,-0.226922$, \\
& & & $-0.082127,0.099221 \mathrm{~T} / \mathrm{m}$ \\
Drift spaces (first eight) & $0.3,1.5,0.5,0.5,6.0$, & System length & $24.65 \mathrm{~m}$ \\
& $0.5,0.5,1.0 \mathrm{~m}$ & & \\
\hline \hline
\end{tabular}

Monte Carlo code G4beamline [22] was used for the multiparticle tracking. The EFP of the lens system is also validated with G4beamline. Figure 3 shows the layout of the G4beamline simulation. Ten quadrupole magnets and four dipole magnets are used in this design.

The parameters for the above lens system are given in Table I. We expect to validate this system on a cyclotron in Institute of Fluid Physics, CAEP.

In the simulation, a parallel incident beam with Gaussian radial distribution (10 $\mathrm{mm}$ in diameter) was used, according to the matching correlation designated in the design procedure. 10,000 protons are simulated for each run.

Figure 4 shows the results of the simulation without energy spread and angular spread. For convenience, momentum is used instead of energy in the simulation.

When there is no energy spread, the profile of the beam is very narrow in the $x$ direction. Since the EFP requires that the particle position depends only on its initial energy, this is just the phenomenon expected.

It is found that the offset of the beam centroid is about 11 $\mathrm{mm}$ with a $2 \%$ momentum offset. This result is in excellent agreement with the simulation by the Transport code, in which $T_{16}$ is found to be $0.554 \mathrm{~m}$.

It is also found that momentum offset will result in beam expansion in the $x$ direction. This is caused by the high

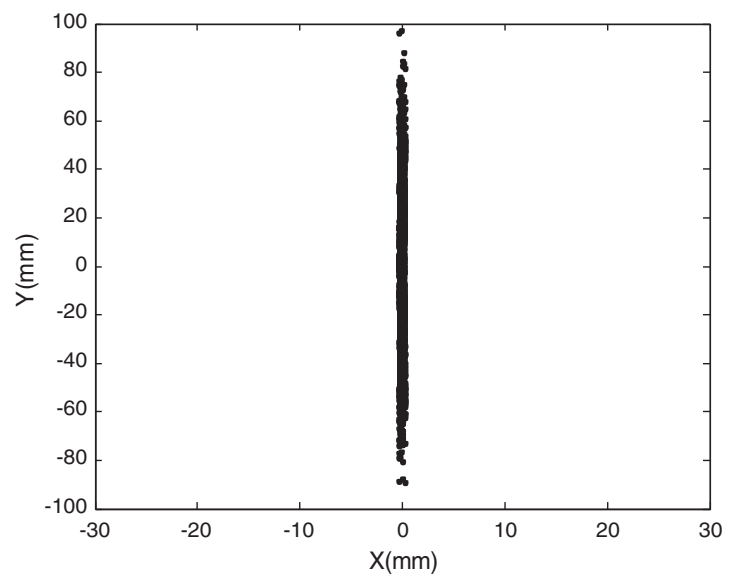

(a) order dynamics of the transport system, and should be further studied in the future.

Figure 5 shows the results with $1 \%$ rms momentum spread. With momentum spread included, the beam size in the $x$ direction is much larger. This confirms the function of energy Fourier plane of the bending magnet.

Besides the energy spread, the beam profile also depends on the initial angle because the coefficient $T_{12}$ couples the angular spread into the EFP [see Eq. (22)]. In this design, $T_{12}$ is calculated to be $1.4 \mathrm{~mm} / \mathrm{mrad}$. Figure 6 shows the results of simulations with rms angular spread of $2 \mathrm{mrad}$.

The energy sorting capability of the lens system is potentially limited by the initial angular spread of the beam, since the transverse beam size at the EFP is a function of both the energy spread and the angular spread. Fortunately, measurements can be taken to reduce $T_{12}$ to a considerably small value (see Sec. IV F), among which the simplest one is to use quadrupole magnets. A tentative design shows that with quadrupole magnets put into the drift spaces between the two pairs of dipole magnets, the coefficient $T_{12}$ can be reduced to a small level around $0.5 \mathrm{~mm} / \mathrm{mrad}$, one third that of the former design.

On the other hand, the coefficient $T_{16}$ is only $0.554 \mathrm{~m}$; a value that may be too small in some cases. It can be increased simply by expanding the distance between the

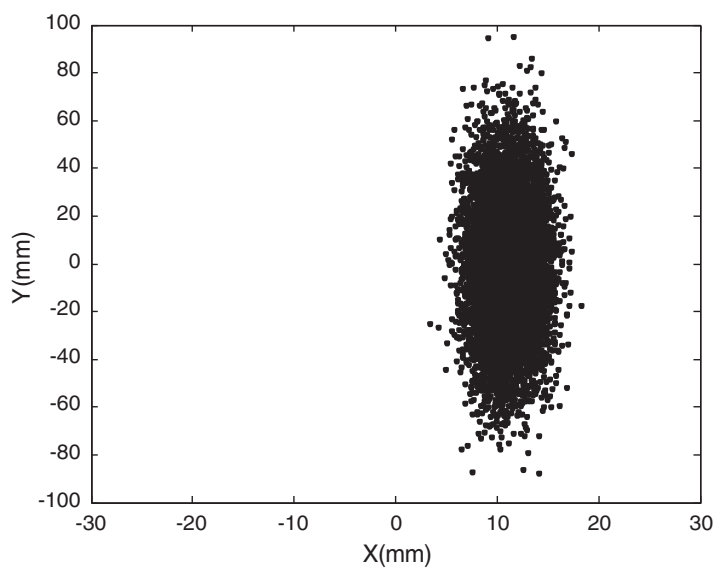

(b)

FIG. 4. Numerical simulation result of beam profile at EFP. (a) without momentum offset. (b)with $2 \%$ momentum offset. 


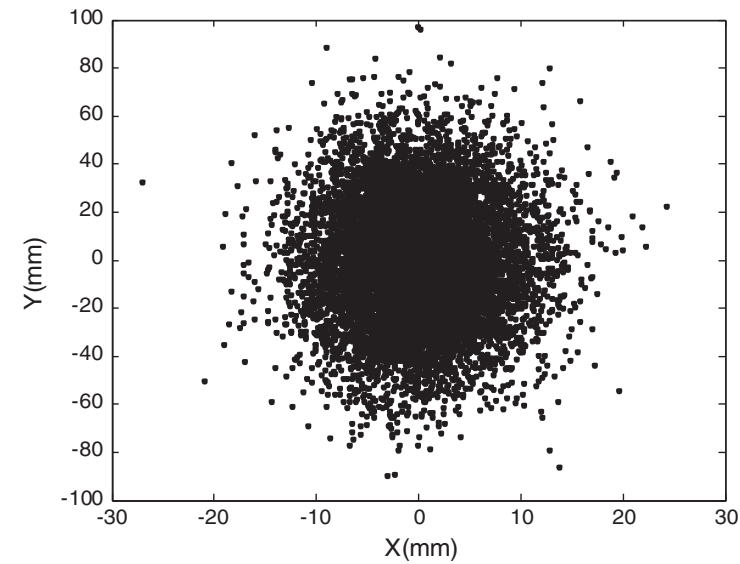

(a)

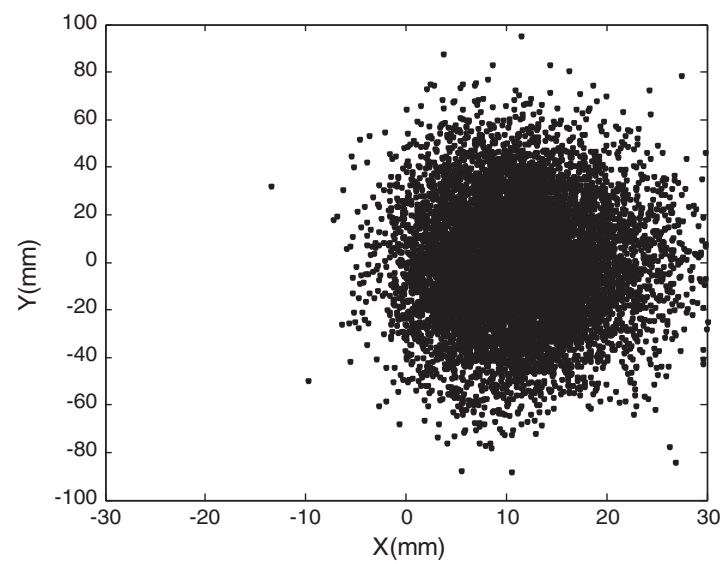

(b)

FIG. 5. Numerical simulation result of beam profile with momentum spread at EFP. (a) no momentum offset, $1 \%$ rms momentum spread. (b) $2 \%$ momentum offset, $1 \%$ rms momentum spread.

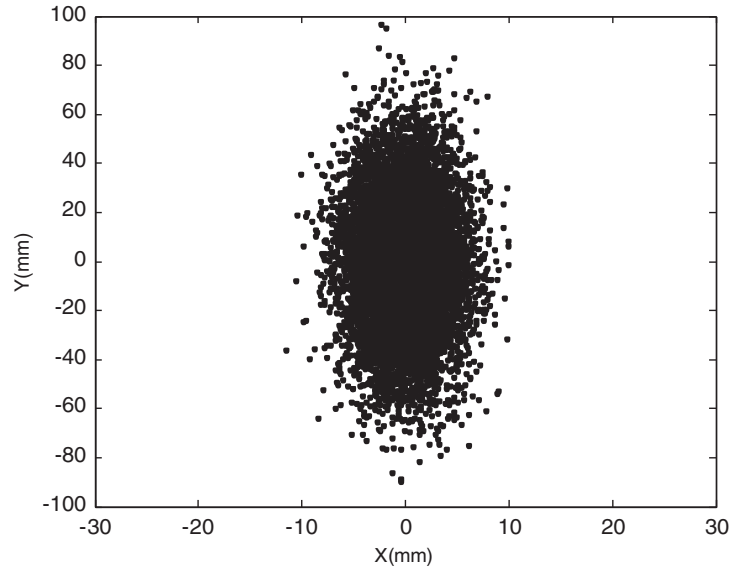

(a)

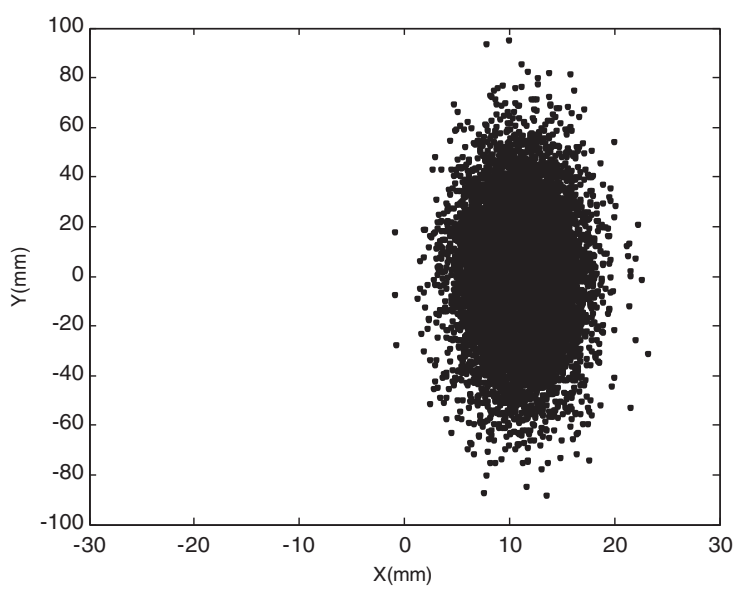

(b)

FIG. 6. Numerical simulation result with angular spread of beam profile at EFP. (a) no momentum offset, $2 \mathrm{mrad}$ rms angular spread. (b) $2 \%$ momentum offset, $2 \mathrm{mrad} \mathrm{rms}$ angular spread.

two parts of the dipole magnet. However, a long drift space may lead to an unacceptable large beam profile. To control the beam profile within a convenient range, quadrupole magnets may also be needed in the beam line.

\section{CONCLUSIONS}

In charged particle radiography, image blur due to multiple Coulomb scattering can be mitigated by using the magnetic lens, which images the particles passing through the object onto the detector. The so-called Zumbro lens makes the proton radiography possible in the diagnosis of a thick object. However, the information of energy loss is useless in the Zumbro lens because the energy loss is the main source of image blur. In light of the concept of the Zumbro lens, an energy-loss imaging lens is proposed in this paper. In the new imaging-lens system, the information of the energy loss becomes useful in forming contrast in the image. The design procedure is presented, and the existence of the energy Fourier plane is proved analytically. A practical design is also demonstrated by numerical simulation. In the future, experimental demonstration of the new imaging-lens system is expected to be carried out on a cyclotron [23] at the Institute of Fluid Physics, CAEP.

\section{ACKNOWLEDGMENTS}

This work was supported by the National Natural Science Foundation of China Grants No. 11205144 and No. 11176001 and by CAEP Developing Foundation Grant No. 2014A0402016. 
[1] A. Koehler, Science 160, 303 (1968).

[2] V. W. Steward and A. Koehler, Science 179, 913 (1973).

[3] V. W. Steward and A. Koehler, Nature (London) 245, 38 (1973).

[4] J. Cookson, Naturwissenschaften 61, 184 (1974).

[5] D. R. Moffett, E. P. Colton, G. A. Concaildi et al., IEEE Trans. Nucl. Sci. 22, 1749 (1975).

[6] K. M. Hanson, J. N. Bradbury, T. M. Cannon, R. L. Hutson, D. B. Laubacher, R. J. Macek, M. A. Paciotti, and C. A. Taylor, Phys. Med. Biol., 26, 965 (1981).

[7] K. M. Hanson, J. N. Bradbury, R. A. Koeppe, R. J. Macek, D. R. Machen, R. Morgado, M. A. Paciotti, S. A. Sandford, and V. W. Steward, Phys. Med. Biol. 27, 25 (1982).

[8] U. Schneider, J. Besserer, P. Pemler, M. Dellert, M. Moosburger, E. Pedroni, and B. Kaser-Hotz, Med. Phys. 31, 1046 (2004).

[9] R. W. Schulte, V. Bashkirov, M. C. Loss Klock, T. Li, A. J. Wroe, I. Evseev, D. C. Williams, and T. Satogata, Med. Phys. 32, 1035 (2005).

[10] C. T. Mottershead and J. D. Zumbro, in Proceedings of the Particle Accelerator Conference, Vancouver, Canada, 1997 (IEEE, Vancouver, 1997), p. 1397.

[11] N. S. P. King, E. Ables, K. Adams et al., Nucl. Instrum. Methods Phys. Res., Sect. A 424, 84 (1999).
[12] T. Mottershead, D. Barlow, B. Blind et al., in Proceedings of the 20th Particle Accelerator Conference, PAC-2003, Portland, OR (IEEE, New York, 2003), p. 702.

[13] C. L. Morris, E. Ables, K. R. Alrick et al., J. Appl. Phys. 109, 104905 (2011).

[14] F. E. Merrill and C. L. Morris, in Proceedings of the 21st Particle Accelerator Conference, Knoxville, TN, 2005 (IEEE, Piscataway, NJ, 2005), p. 1715.

[15] R. M. Barnett, C. D. Carone, D. E. Groom et al., Phys. Rev. D 54, 1 (1996).

[16] H. A. Bethe, Phys. Rev. 89, 1256 (1953).

[17] D. Groom, Energy loss in matter by heavy particles. PDG93-06, Particle Data Group, 1993.

[18] J.W. Goodman, Introduction to Fourier Optics (Mc Graw-Hill, New York, 1968).

[19] G. Geloni, E. Saldin, and E. Schneidmiller, Opt. Commun. 276, 167 (2007).

[20] Lv. Jianqin, The Optics of Charged Particle Beams (High Education Press, Beijing, China, 2004), p. 158 (in Chinese).

[21] PSI Graphic Transport Framework by U. Rohrer based on a CERN-SLAC-FERMILAB version by K. L. Brown et al.

[22] T. Roberts, G4Beamline user's guide, available at http:// g4beamline.muonsinc.com.

[23] W. Tao, Y. Guojun, and H. Xiaozhong, High Power Laser Part. Beams 24, 2193 (2012) (in Chinese). 